\title{
Corioangioma placentario: reporte de un caso en la Unidad de Patología del Hospital San Juan de Dios E.S.E. Rionegro-Antioquia
}

\section{Placental chorioangioma: case report in the Pathology Unit of the San Juan de Dios E.S.E. Hospital in Rionegro-Antioquia}

\author{
Juan Esteban Torres-Correa ${ }^{1}$, Mayra Alejandra Sánchez-Montoya², \\ Jairo Sandoval-Sánchez ${ }^{3}$, John Fredy Castro-Álvarez ${ }^{4}$
}

\begin{abstract}
Resumen. Dentro de las neoplasias no trofoblásticas gestacionales de tipo vascular, el corioangioma es la más común. Se caracteriza por presentar una proliferación exagerada de estructuras vasculares, con células endoteliales y vellosidades coriónicas que generan una alteración de los vasos sanguíneos tanto arteriales como venosos. Tiene una incidencia menor al $1 \%$, con tasas de mortalidad perinatal aproximadas entre $18 \%$ a $40 \%$. Se presenta el caso de un corioangioma placentario diagnosticado a las 26 semanas de gestación en una paciente de 21 años de edad sin controles prenatales previos, el cual generó un lóbulo accesorio de $7 \mathrm{~cm}$ con tinción positiva en el análisis inmunohistoquímico para anticuerpos antimúsculo liso y CD34, y negativa para Ki67. El corioangioma desencadenó complicaciones en el embarazo, con parto pretérmino, hidropesía fetal y retardo en el crecimiento intrauterino, que llevaron a la muerte fetal. La falta de control prenatal en nuestra paciente impidió que se detectaran las anomalías placentarias oportunamente, con un desenlace fatal para el feto. Un control prenatal adecuado con seguimiento ecográfico puede evitar eventos fatales como el presentado. El análisis macroscópico y microscópico de la placenta puede favorecer la identificación de los casos y el aporte a las estadísticas de incidencia y mortalidad.
\end{abstract}

Palabras clave: hemangioma, inmunohistoquímica, neoplasias trofoblásticas, embarazo, muerte fetal.

\footnotetext{
${ }^{1}$ Médico General. Facultad de Ciencias de la Salud, Corporación Universitaria Remington. Medellín, Colombia.

${ }^{2}$ Médica General. Facultad de Ciencias de la Salud, Corporación Universitaria Remington. Medellín, Colombia.

${ }_{3}^{3}$ Médico, Especialista en Patología. Unidad de Patología del Hospital San Juan de Dios E.S.E. Rionegro. Grupo de Investigación en Neurociencias y Envejecimiento, Facultad de Ciencias de la Salud, Corporación Universitaria Remington. Medellín, Colombia.

${ }^{4}$ Microbiólogo y Bioanalista. MSc y PhD en Biología. Grupo de Investigación en Neurociencias y Envejecimiento , Facultad de Ciencias de la Salud, Corporación Universitaria Remington. Medellín, Colombia. E-mail: john.castro@uniremington.edu.co.

Conflicto de interés: los autores declaran que no tienen conflicto de interés.

Medicina \& Laboratorio 2020;24:325-332. https://doi.org/10.36384/01232576.340

Recibido el 20 de diciembre de 2019; aceptado el 11 de mayo de 2020. Editora Médica Colombiana S. A., 2020
} 
Abstract. Among vascular gestational nontrophoblastic tumors, chorioangioma is the most common. It is characterized by an exaggerated proliferation of vascular structures, with endothelial cells and chorionic villi that generate abnormalities of both arterial and venous blood vessels. It has an incidence of less than 1\%, with perinatal mortality rates between $18 \%$ to $40 \%$. We present the case of a 21 -year-old patient without previous prenatal controls, with a placental chorioangioma diagnosed at 26 weeks of gestation that formed a $7 \mathrm{~cm}$ accessory lobe, with positive staining during immunohistochemical analysis for smooth anti-muscle antibodies and CD34, and negative for Ki67. The placental chorioangioma triggered complications during pregnancy, with preterm delivery, hydrops fetalis, and intrauterine growth retardation leading to fetal death. The lack of prenatal control in our patient prevented an early detection of placental abnormalities with a fatal outcome for the fetus. Adequate prenatal control with ultrasound monitoring can avoid fatal events such as the one presented. Macroscopic and microscopic analysis of the placenta can help identify cases and contribute to incidence and mortality statistics.

Keywords: hemangioma, immunohistochemistry, trophoblastic neoplasms, pregnancy, fetal death.

\section{Introducción}

Las neoplasias no trofoblásticas gestacionales de tipo vascular son patologías de escasa presentación y fueron descritas por Clarke en 1798 [1]. Dentro de estas neoplasias el tumor más común es el corioangioma, el cual se caracteriza por presentar una proliferación exagerada de estructuras vasculares con células endoteliales y vellosidades coriónicas, que generan una alteración de los vasos sanguíneos tanto arteriales como venosos [2]. Históricamente se le ha dado diferentes nombres, incluyendo hemangioma, angioma placentario, corangioma, mesenquimoma, hamartoma vascular de la placenta, fibroma, mixoma o corioma, entre otros.

El corioangioma placentario tiene una incidencia menor al $1 \%$ en los embarazos, con tasas de mortalidad perinatal estimadas que varían entre 18\% a $40 \%$ [3]. Estos datos estadísticos podrían ser mayores si la placenta se estudiara tanto macroscópica como microscópicamente de manera rutinaria [4-6], ya que la ma- yoría de los tumores tienen tamaños menores a $5 \mathrm{~cm}$ y pasan desapercibidos por el médico al momento de la revisión de la placenta [4]; además, estos tienen pocas complicaciones durante la gestación. Cuando estos tumores alcanzan un tamaño mayor de $5 \mathrm{~cm}$ pueden causar diferentes situaciones clínicas en la madre, como metrorragia, parto pretérmino, polihidramnios y preeclampsia, entre otros, y pueden causar complicaciones graves en el feto, como anemia fetal, hidrops no aloinmunitario e insuficiencia cardiaca fetal, llegando incluso a comprometer la vida de este [2-8].

En este artículo se reporta el caso de un corioangioma placentario gigante, diagnosticado en el laboratorio de patología del Hospital San Juan de Dios E.S.E de Rionegro-Antioquia, que tuvo como consecuencia la muerte fetal.

\section{Caso clínico}

Paciente femenina de 21 años de edad, nulípara, con un embarazo de 26 sema- 
nas por fecha de la última menstruación, sin controles prenatales previos, quien consultó al servicio de urgencias del Hospital San Juan de Dios E.S.E de Rionegro por presentar dolor abdominal de 8 horas de evolución, acompañado de secreción vaginal amarillenta. Al examen físico presentó signos vitales estables con una altura uterina de $23 \mathrm{~cm}$, fetocardia positiva, borramiento del cuello uterino del $70 \%$ y dilatación de $5 \mathrm{~cm}$. Se le solicitó ecografía donde se evidenció feto en posición podálica y una placenta corporal anterior, además de una frecuencia cardiaca fetal de 144 latidos por minuto.

Se decidió iniciar trabajo de parto. Se recibió un feto con talla de $30 \mathrm{~cm}$, perímetro cefálico de $21 \mathrm{~cm}$, perímetro torácico de $18 \mathrm{~cm}$, tórax con petequias y un abdomen distendido con hepatoesplenomegalia. Se diagnosticó hidropesía fetal, aunque también se sospechó un síndrome dismórfico, además de ser un prematuro extremo y sin signos vitales. La madre presentó retención placentaria, por lo que se decidió extracción manual; al momento de la visualización de dicha placenta, se observaron estructuras ve- siculares que sugirieron la presencia de una mola hidatiforme por su apariencia de racimo de uvas (figura 1). La paciente se complicó con sangrado abundante, lo cual desencadenó un código rojo, siendo necesaria la administración de líquidos y transfusión sanguínea, lográndose la estabilización de la paciente. La placenta y el feto se remitieron al servicio de Patología. La descripción macroscópica confirmó un feto de sexo femenino con una longitud céfalo-caudal de $17 \mathrm{~cm}$, longitud total de $30 \mathrm{~cm}$, peso de $289 \mathrm{~g}$, perímetro cefálico de $22 \mathrm{~cm}$, perímetro torácico de $20 \mathrm{~cm}$ y perímetro abdominal de $21 \mathrm{~cm}$, con retardo del crecimiento intrauterino (RCIU) para la edad gestacional. El feto presentó apariencia eritematosa tipo arlequín, con esfacelación en extremidades inferiores, microcéfalo y con edema en región cervical, principalmente en la nuca; los demás tejidos no presentaron alteraciones histológicas. En el cordón se observaron tres estructuras vasculares (dos arterias y una vena). A la apertura de cavidades toraco-abdominales, se observó líquido cetrino y algunos órganos en lisis, los cuales se encontraron en su posición anatómica. Al examen macroscópico se

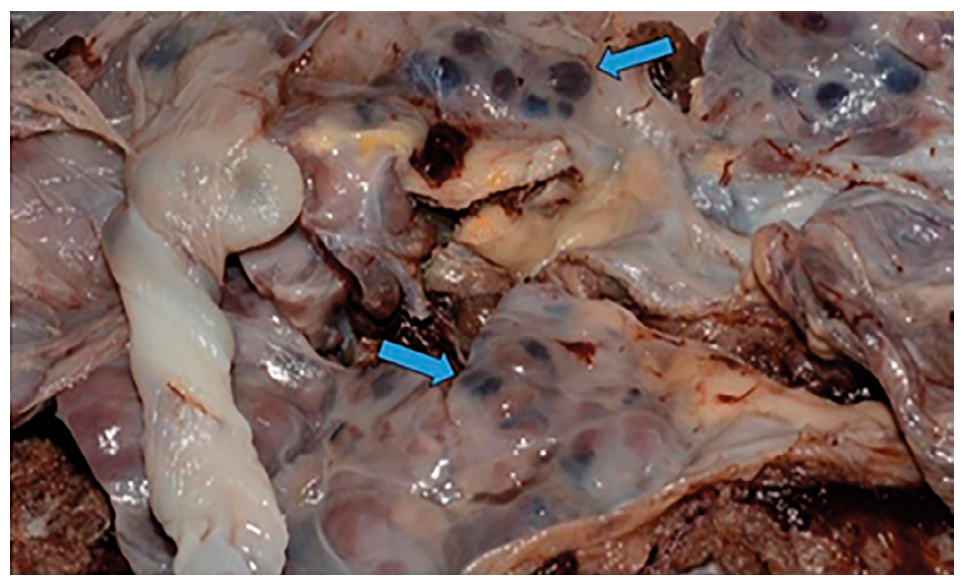

Figura 1. Vista macroscópica de la placenta. Cara fetal de la placenta en donde se observan estructuras vesiculares sugestivas de enfermedad molar. 
evidenció hepatoesplenomegalia y un hígado con aspecto micronodular, y al examen microscópico el hígado mostró una eritropoyesis extramedular. En la disección cardiaca se observó una comunicación aurículo-ventricular e interauricular de 1,5 $\mathrm{mm}$. Se reportó un peso de $29 \mathrm{~g}$ para el bazo, con bazo accesorio, ambos hemorrágicos.

La placenta presentó múltiples fragmentos lacerados los cuales en conjunto alcanzaron un peso de $170 \mathrm{~g}$ (figura 2). El cordón presentó una ruptura en su inserción, la cara fetal placentaria fue de color blanquecino con un lóbulo accesorio de aproximadamente 7 $\mathrm{cm}$. Al momento del corte se observó una coloración parda hemorrágica con un sangrado mayor a lo habitual. En la descripción microscópica se evidenció una neoplasia vascular placentaria no trofoblástica de estirpe mesenquimal con predominio angiomiomatoso y múltiples vasos de tipo capilar de paredes delgadas, las cuales estaban tapizadas por endotelio donde era posible observar núcleos aumentados de tamaño con una mayor condensación cromatínica, y con glóbulos rojos nucleados en su interior (figura 3). El resto de la placenta correspondía a vellosidades placentarias inmaduras del segundo trimestre del embarazo, algunas de tamaño intermedio y otras de gran tamaño, con estroma laxo. No se observaron cisternas o cordón con vasos obliterados; estos hallazgos descartaron enfermedad trofoblástica.

Debido a los hallazgos de neoplasia vascular encontrados con la coloración de hematoxilina y eosina, se realizó un análisis por inmunohistoquímica para confirmar la naturaleza endotelial de la lesión y su índice proliferativo. Con base en los resultados positivos para CD34 (figura 4A) y anticuerpos antimúsculo liso (SMA) (figura 4B), en una placenta lacerada inmadura con un índice proliferativo de 0 (Ki67 negativo) (figura 4C), el reporte de patología concluyó que el diagnóstico correspondía a un corioangioma placentario (tumor no trofoblástico de estirpe vascular). La paciente fue dada de alta en el posparto sin complicaciones asociadas.

\section{Discusión}

Los tumores placentarios se dividen en trofoblásticos y no trofoblásticos; dentro de los no trofoblásticos el más

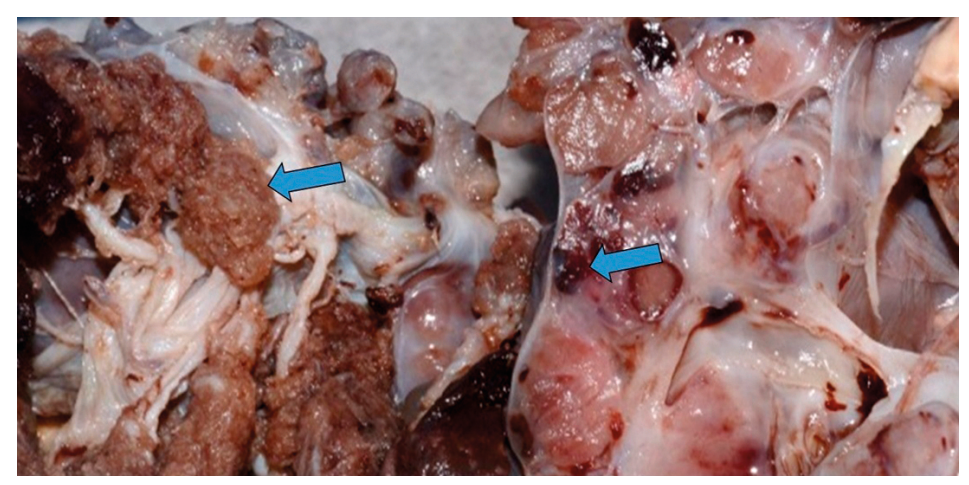

Figura 2. Macroscopía tumoral. Al lado izquierdo de la imagen se visualiza la placenta lacerada con trofoblasto usual, y al lado derecho, la zona tumoral con aumento de la vasculatura y hemorragias. 


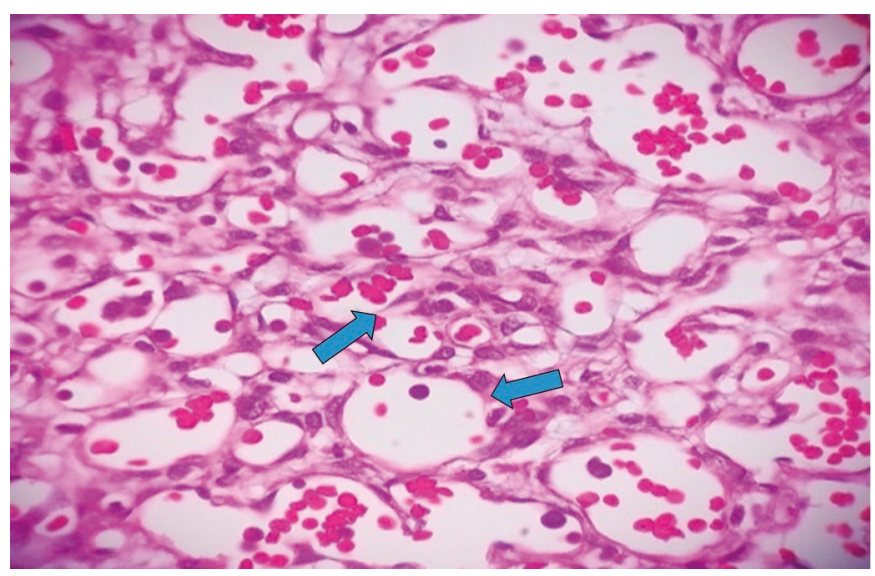

Figura 3. Microscopía de la neoplasia. Se observan estructuras vasculares capilares con paredes delgadas tapizadas por endotelio, núcleos aumentados de tamaño y en su interior eritrocitos. Hematoxilina y eosina (40x).

común es el corioangioma [9]. Según la literatura, la frecuencia del corioangioma es de aproximadamente $1 \%$ en las placentas sometidas a estudio anatomopatológico $[2,4]$. Hasta el momento, en Colombia hay cuatro reportes de esta patología entre los años 1990 a 2019 [10-13]. Los estudios de este tumor se remiten al año 1798, cuando fue descrito el primer caso de corioangioma por Clarke [1]. Actualmente el diag- nóstico se realiza por ultrasonografía, convirtiéndose este en el principal método diagnóstico dependiendo del tamaño del tumor, en donde es posible observar una masa de consistencia sólida ubicada en el interior de la placenta, con una ecogenicidad irregular [14]. A través del Doppler a color se pueden observar los vasos de alimentación que ingresan a la masa placentaria y la vascularización difusa peritumoral [7]. A
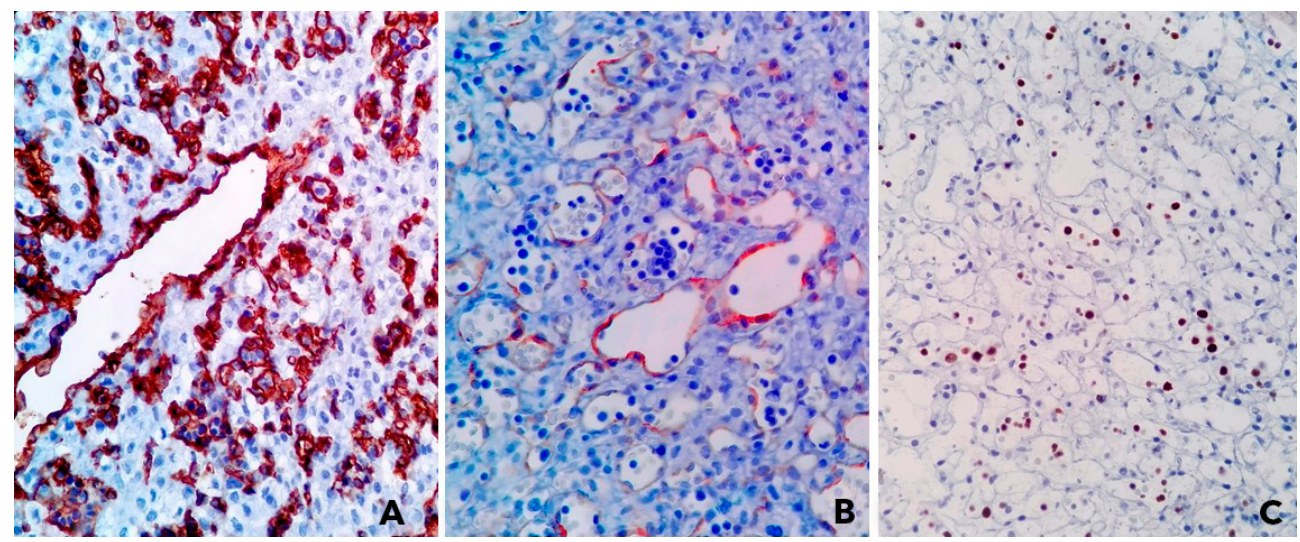

Figura 4. Análisis inmunohistoquímico confirmatorio de la neoplasia, donde se observa tinción positiva para CD34 (A) y SMA (B) que evidencia vasos constitutivos de la neoplasia e índice proliferativo de 0 con un Ki67 negativo (C) (40x). 
pesar de que la ultrasonografía se considera como el estándar de oro para el diagnóstico, pueden surgir dificultades al diferenciar subtipos celulares y degenerativos de corioangioma donde la vascularización es menos evidente [15]. Existen otros métodos diagnósticos anteparto, además de la ecografía, por los cuales se puede llegar al diagnóstico de esta patología; entre ellos, la medición de la alfa fetoproteína tanto en la madre como en el feto [2,5], y desde hace aproximadamente 10 años, el uso de la técnica de ecografía VOCAL (del inglés, Virtual Organ Computer-Aided Analysis), la cual consiste en evaluar el crecimiento o involución de los vasos a nivel de la placenta, observándose así el comportamiento tumoral $[12,16]$. Debido a que la paciente nunca acudió a controles prenatales, no fue posible hacer un diagnóstico mediante estudios imagenológicos, los cuales son de gran importancia durante la gestación.

El corioangioma placentario se caracteriza por tener alteraciones a nivel arterial y venoso, debido a una proliferación exagerada de las estructuras vasculares a nivel del endotelio y de las vellosidades coriónicas, que generan una ubicación en el margen placentario o cerca a la placa coriónica $[5,9,10]$. Es de importancia resaltar que, pese a su alta capacidad de división celular, sigue siendo un tumor no trofoblástico de características benignas; sin embargo, tiene serias complicaciones tanto para la madre como para el recién nacido, llegando a comprometer la vida de ambos [4,9]. La mayoría de estos tumores son de una presentación única y se diagnostican frecuentemente mediante ecografía $[9,17]$; aunque en este caso clínico no se identificó hasta el posparto, es posible que la tumoración de $7 \mathrm{~cm}$ fuera visible en imágenes diagnósticas, lo cual hace necesario no solo que las mujeres gestantes acudan a los controles prenatales, sino que también exista personal entrenado para detectar este tipo de tumores poco frecuentes mediante pruebas imagenológicas anteparto.

Más del $50 \%$ de los corioangiomas son de tamaño pequeño ocasionando pocos síntomas tanto en la madre como en el feto, pero cuando su tamaño alcanza más de $5 \mathrm{~cm}$ se describen como "gigantes" $[2,4]$. Estas neoplasias pueden llevar a que la madre desarrolle preeclampsia, metrorragias, desprendimiento prematuro de placenta, parto pretérmino o polihidramnios $[4-8,18]$. En el feto se presentan alteraciones a nivel cardiovascular (falla cardiaca congestiva y cardiomegalia), hematógena (coagulación intravascular diseminada y anemia), alteraciones en el crecimiento (RCIU, prematuridad) y muerte fetal, dependiendo del tamaño de la neoplasia en la placenta o de su vascularización, ya que este es un factor pronóstico para el corioangioma placentario $[4-8,18]$. Los corioangiomas no vascularizados tienden a no asociarse con complicaciones, mientras que los tumores vascularizados pueden predisponer a las complicaciones típicas del corioangioma [15]. La fisiopatología se explica por el cambio que sufren las vellosidades placentarias y la relación con los bajos niveles de oxígeno en las unidades placentarias y fetales, haciendo que se genere un aumento del tamaño del cordón umbilical y la placenta debido a la retención de líquido; estos cambios son observables por ecografía, siendo de gran importancia para un diagnóstico eficaz $[2,18,19]$.

En la actualidad para hacer un diagnóstico acertado de esta patología se cuenta con diferentes marcadores inmunohistoquímicos, con la finalidad de corroborar los hallazgos macros- 
cópicos y microscópicos en aquellos casos en donde se sospeche esta entidad; el diagnóstico definitivo se establece mediante el examen histológico del tumor [15]. A continuación, se describen los principales marcadores y su respectiva importancia:

- CD34: marcador de células progenitoras hematopoyéticas y células endoteliales de uso frecuente. Es importante para la diferenciación de los hemangiopericitomas y de los hemangiomas [20].

- Ki67: es una proteína que se expresa en las fases G1, S, G2 y M del ciclo celular. Se cataboliza rápidamente al final de la fase $M$ y no es detectable en células que se encuentren en G0 ni en G1 temprana. Su positividad indica que es una lesión de tipo maligno [21].

- $\boldsymbol{\beta}$-catenina: proteína codificada por el gen CTNNB1, que coordina la adhesión célula-célula y la transcripción génica. Se ha encontrado positividad en uno de cada tres casos de corangiocarcinoma [22].

- SMA: anticuerpos contra la actina del músculo liso. Este marcador tiene alta especificidad para el componente de actina en el citoesqueleto. Los anticuerpos pueden ser detectados mediante inmunofluorescencia indirecta, permitiendo diferenciar enfermedades autoinmunes, afectando principalmente el tejido hepático, como ocurre en la hepatitis autoinmune y en la cirrosis biliar primaria [23].

Se recalca la importancia de acudir al control prenatal con el fin de identificar cualquier signo temprano de compromiso fetal. Los embarazos que presentan masa placentaria grande deben someterse a un monitoreo fetal intensivo, por lo que se sugiere evaluación fetal cada 1 a 2 semanas [6], en tanto que para los corioangiomas pequeños se recomienda el monitoreo cada 3 a 4 semanas [15]. La falta de control prenatal en nuestra paciente impidió que se detectaran las anomalías placentarias oportunamente, con un desenlace fatal para el feto. Es también importante reportar los casos que se encuentren de corioangioma placentario, con la finalidad de contribuir al conocimiento y estimar las tasas de morbilidad y mortalidad maternofetal reales. Además, es trascendente la evaluación cuidadosa de las placentas, y se reitera la importancia del estudio macroscópico y microscópico por parte de Patología, con la finalidad de poder dar una explicación más precisa a los familiares y al personal asistencial de lo que pudo haber llevado a la pérdida o complicación del embarazo.

\section{Referencias}

1. Clarke J, Banks J. Account of a tumour found in the substance of the human placenta. Philos Trans Royal Soc 1798;88:361-368.

2. Fan M, Skupski DW. Placental chorioangioma: literature review. J Perinat Med 2014;42:273279. https://doi.org/10.1515/jpm-2013-0170.

3. Lim FY, Coleman A, Polzin W, Jaekle R, Habli M, Van Hook J, et al. Giant chorioangiomas: perinatal outcomes and techniques in fetoscopic devascularization. Fetal Diagn Ther 2015;37:18-23. https://doi. org/10.1159/000363600.

4. Guschmann $M$, Henrich $\mathbf{W}$, Entezami M, Dudenhausen JW. Chorioangioma: new insights into a well-known problem. I. Results of a clinical and morphological study of 136 cases. J Perinat Med 2003;31:163-169. https://doi. org/10.1515/jpm.2003.022.

5. Wu Z, Hu W. Clinical analysis of 26 patients with histologically proven placental chorioangiomas. Eur J Obstet Gynecol Reprod Biol 2016;199:156-163. https://doi.org/10.1016/j. ejogrb.2015.12.009. 
6. Buca D, lacovella C, Khalil A, Rizzo G, Sirotkina M, Makatsariya A, et al. Perinatal outcome of pregnancies complicated by placental chorioangioma: systematic review and meta-analysis. Ultrasound Obstet Gynecol 2020;55:441449. https://doi.org/10.1002/uog.20304.

7. Zanardini C, Papageorghiou A, Bhide A, Thilaganathan B. Giant placental chorioangioma: natural history and pregnancy outcome. Ultrasound Obstet Gynecol 2010;35:332-336. https://doi.org/10.1002/uog.7451.

8. Liu H, Gu W, Li X. Natural history and pregnancy outcome in patients with placental chorioangioma. J Clin Ultrasound 2014;42:74-80. https://doi.org/10.1002/jcu.22101.

9. Guschmann M, Henrich W, Dudenhausen JW. Chorioangiomas: new insights into a well-known problem. II. An immuno-histochemical investigation of 136 cases. J Perinat Med 2003;31:170175. https://doi.org/10.1515/jpm.2003.023.

10. Pérez GG, Sierra AJ, Rangel NE, Fuentes PJ. Corangioma placentario: enfoque clínico-patológico de un caso descrito en Colombia. Ginecol Obstet Mex 2013;81:109-114.

11. Sarmiento-Rodríguez A, Quijano-García FE, Puccini-Santamaría G, Rodríguez-Acosta N, Victoria-Gómez PA. Corioangioma placentario: Reporte de caso y revisión de la literatura. Rev Colomb Obstet Ginecol 2008;59:57-61.

12. Acosta-Cajiao E, Acosta-Lleras E, Amaya L, Navas G. Corioangioma diagnóstico anteparto e implicaciones fisiopatológicas. Rev Colomb Obstet Ginecol 1990;41:233-237. https://doi. org/10.18597/rcog. 973.

13. Ramírez-Cárdenas A, Licona-Venegas EE, Licona-Meníndez RD. Corioangioma placentario gigante. Reporte de un caso. Ginecol Obstet Mex 2018;86:692-698.

14. Kurjak A, Chervenak FA. Ecografía en Obstetricia y Ginecologia. 2da ed. Buenos Aires, Argentina: Editorial Médica Panamericana S.A.; 2009. p. 1010. ISBN 9789500609135.

15. Abdalla N, Piórkowski R, Stanirowski $P$, Pazura M, Cendrowski K, Sawicki W. Can ultra- sound be helpful in selecting optimal management methods for pregnancies complicated by placental non-trophpblastic tumors? J Ultrason 2017;17:116-122. https://doi.org/10.15557/ JoU.2017.0017.

16. Domingo ID, Coronado Martin P, Badillo CA, Rincón J, Montalvo J, Cristobal I, et al. Placental chorangioma: Prenatal ultrasound diagnostic. Tokoginecol Pract 2001;60:272-275.

17. Gonzalo I, Martínez-Guisasola J, Martín MA, Rozada P, Klabili M, Fernández G. Corioangioma placentario gigante. Diag Pren 2011;22:136-138. https://doi.org/10.1016/j.diapre.2011.11.002.

18. Sirotkina M, Douroudis $K$, Westgren M, Papadogiannakis N. Association of chorangiomas to hypoxia-related placental changes in singleton and multiple pregnancy placentas. Placenta 2016;39:154-159. https://doi.org/10.1016/j.placenta.2016.01.020.

19. Ogino S, Redline RW. Villous capillary lesions of the placenta: distinctions between chorangioma, chorangiomatosis, and chorangiosis. Hum Pathol 2000;31:945-954. https://doi.org/10.1053/ hupa.2000.9036.

20. Stuar LN. CD markers, CD34. Michigan, USA: PathologyOutlines.com; 2019. Acceso 21 de noviembre de 2019. Disponible en http:// www.pathologyoutlines.com/topic/cdmarkerscd34.html.

21. Roychowdhury M. Stains Ki67/MIB1. Michigan, USA: PathologyOutlines.com; 2019. Acceso 10 de noviembre de 2019. Disponible en http://www. pathologyoutlines.com/topic/stainski67.html.

22. Pernick N. Stains, beta-catenin. Michigan, USA PathologyOutlines.com; 2018. Acceso 12 de noviembre de 2019. Disponible en https:// www.pathologyoutlines.com/topic/stainsbetacatenin.html.

23. Johnson G. Stains, actin alpha smooth muscle type. Michigan, USA: PathologyOutlines.com; 2018. Acceso 18 de noviembre de 2019. Disponible en http://www.pathologyoutlines.com/topic/ stainsalphasmoothmuscleactin.html. 